\title{
Vergleich der Bestimmung \\ von Leukozytenpopulationen am Coulter VCS mit der mikroskopischen Differenzierung
}

\author{
Comparison of Performance for Leukocyte Differential Counting of Coulter VCS with Mi- \\ croscopical Analysis
}

\author{
J. Ennen, R. Bäurle, H. Schriewer \\ Zentrallaboratorium des Kreiskrankenhauses, Lûdenscheid
}

\begin{abstract}
Zusammenfassung:
Der cytometrisch-volumetrisch arbeitende Differentialblutbildautomat VCS von Coulter wurde hinsichtlich seiner Präzision, seiner Linearität und möglicher Verschleppungseffekte untersucht. Der VCS zeigt eine hohe Präzision bei den Zellpopulationen der Neutrophilen und der Lymphozyten, bei den Eosinophilen und Monozyten eine gute Präzision sowie eine mäßige Präzision bei den basophilen Granulozyten.
\end{abstract}

Analytisch relevante Verschleppungseffekte machen sich nur in Extremsituationen bemerkbar (z. B. Messung einer Probe mit 400 Leukozyten im Anschluß an eine Probe mit 90000 Leukozyten).

Verdünnungsexperimente mit homologem EDTA-Plasma ergaben keine nennenswerten Abweichungen von der Linearität.

An drei Patientenkollektiven, I normale Blutbilder $(N=96)$, II reaktiv linksverschobene Blutbilder $(N=283)$, III onkologische Blutbilder $(N=96)$, wurde die Differenzierung von Leukozyten durch cytometrisch-volumetrische Analyse am VCS von Coulter vergleichend mit der visuellen Mikroskopie von Blutausstrichen durchgeführt (pro Ausstrich wurden 250 Leukozyten ausgezählt).

Unsere Untersuchung zeigt folgende Ergebnisse:

- Die Auswertung nach Passing und Bablok zeigt für Kollektiv I und Kollektiv Il gute bis sehr gute Korrelationen für die Zellpopulationen Lymphozyten, neutrophile Granulozyten, eosinophile Granulozyten und für Monozyten. Bei den basophilen Granulozyten konnte eine lineare Beziehung zwischen automatisiert und visuell erstelltem Differentialblutbild nicht nachgewiesen werden, da die statistische Zählgenauigkeit beim visuell erstellten Differentialblutbild mit nur 250 Zellen bei gleichzeitig kleiner Zellpopulation nicht ausreicht.

- Die Sensitivität des VCS in bezug auf Erkennung von reaktiv-linksverschobenen Blutbildern (Kollektiv II) liegt etwa bei $99 \%$.

- Hochpathologische, onkologische Blutbilder werden, unter Berücksichtigung der Gesamtleukozytenzahl, mit einer Sensitivität von $84,4 \%$ als pathologisch indiziert.

\section{Schlüsse/wörter:}

Leukozyten - Differentialblutbild - VCS von Coulter - neutrophile Granulozyten - eosinophile Granulozyten

- basophile Granulozyten - Monozyten - Lymphozyten

\section{Summary:}

The cytometric-volumetric analyser for leucocyte differential counting VCS was evaluated in respect of precision, linearity and carry over effects. The precision for granulocyte and lymphocyte counting was very good, for eosinophils and monocytes quite good and moderate for basophils. Carry over effects were observed only under extrem analytical conditions (for example: Measuring of a sample with 400 leukocytes total in conection with a blood sample with 90.000 leukocytes).

No remarkable deviation from linearity was found in dilution experiments using homolog EDTA-plasma as diluent.

The leukocyte differential counting of the cytometric-volumetric analyser VCS, Coulter was compared to microscopical analysis of three different collectives of samples, I normal blood samples ( $N=96)$, II blood samples with a left shift representing the acute response to inflammation (left shift defined as increased count of band nuclei in granulocytes $(>8 \%)(N=283)$, III oncologic blood samples (for example leukemias) $(N=96)$. To obtain a statistically acceptable white cell counting in visuell microscopy 250 leukocytes were differentiated in blood smears by two investigators each.

The findings of this evaluation are:

- The linear regression analysis according to Passing/Bablok indicates a good or even a very good correlation for lymphocytes, granulocytes, eosinophils and monocytes. Contrary to these findings no correlation was found for basophilic granulocytes, because the statistical exactness of microscopical analysis (250 cells/ blood smear) is not sufficient for the small population of basophils. 

- The sensitivity of VCS, Coulter to recognize blood samples with a left shift (collective II) is very good, ex-
ceeding almost $99 \%$.

- In regard to total leukocyte counting the sensitivity of the VCS, Coulter to indicate oncologic samples by "flagging " is about $84.4 \%$.

Keywords:

Leukocytes - differential white cell counting - VCS Coulter - granulocytes - eosinophils - basophils - monocytes - lymphocytes

\section{Einleitung}

Differentialblutbilder gehören auch heute noch zu den Grundpfeilern der Diagnostik von Erkrankungen des blutbildenden Systems. Sie erlauben ferner wichtige Rũckschlũsse auf systemische Organerkrankungen. Die traditionelle mikroskopische Differenzierung ist allerdings zeit-, personal- und damit kostenautwendig. Da in der Regel nur 100 Zellen ausgewertet werden, ist das Ergebnis der mikroskopischen Blutbilddifferenzierung außerdem mit einer hohen statistischen Unsicherheit behaftet (1-3): Von 5 gefundenen Zellen einer Art können sich unter Berücksichtigung des 95\%-Konfidenzintervalls zwischen $1 \%$ und $12 \%$ dieser Zellen im Blut befinden (Abb. 1).

Im Zuge einer zunehmenden Rationalisierung der Laboratoriumsmedizin wurden in den letzten Jahren Geräte entwickelt, die eine Mechanisierung des Differentialblutbildes ermöglichen (4-13).

Der besondere Vorteil dieser Geräte ist die ca. 10fache Steigerung der Präzision der Blutbilddifferenzierung, die sich in erster Linie bei kleinen Zellpopulationen wie eosinophile und basophile Granulozyten sowie bei den Monozyten auswirkt. Dieser Gewinn an statistischer Genauigkeit ist vor allem bei Verlaufskontrollen einer Erkrankung anhand von zahlenmäßigen Veränderungen von Zellen des Blutbildes von großer Wichtigkeit (z. B. Therapiekontrolle bei Zytostase). Beim Gerät VCS der Firma Coulter handelt es sich um einen Automaten, der aus der cytometrisch-volumetrischen Analyse einer EDTA-Voll- blutprobe ein vollständiges automatisiertes DifferentialBlutbild erstellen kann. Analyse und Klassifikation der Zellen hängen von drei Meßprinzipien ab:

- individuelles Zellvolumen (Messung eines sich proportional zum Zellvolumen verhaltenden Widerstandes bei Einsatz eines niederfrequenten Stromes)

- Hochfrequenzleitfähigkeit (spiegelt innere physikalische und chemische Beschaffenheit einer Zelle wider)

- Oberflächenlichtstreuung an Partikeln (spiegelt externe Zellstrukturen wider).

Ziel unserer Untersuchung war es, festzustellen, ob der Differential-Blutbildautomat VCS von Coulter

- normale Zellen des Differentialblutbildes (neutrophile, eosinophile und basophile Granulozyten, Lymphozyten und Monozyten) zuverlässig erkennt

- reaktiv-linksverschobene Blutbilder (8 stabkernige Granulozyten und mehr oder jüngere Vorstufen) richtig erkennt und

- im visuellen Differentialblutbild als pathologisch eingestufte Blutbilder von Patienten mit gesicherten malignen Erkrankungen des blutbildenden Systems zuverlässig signalisiert.

\section{Material und Methoden}

Die Untersuchung wurde in drei Abschnitten durchgeführt:

Abschnitt I: 96 nichtpathologische Blutbilder mit normalen'Leukozytenzahlen aus der täglichen Routine des Zentrallaboratoriums wurden am VCS, Coulter gemessen

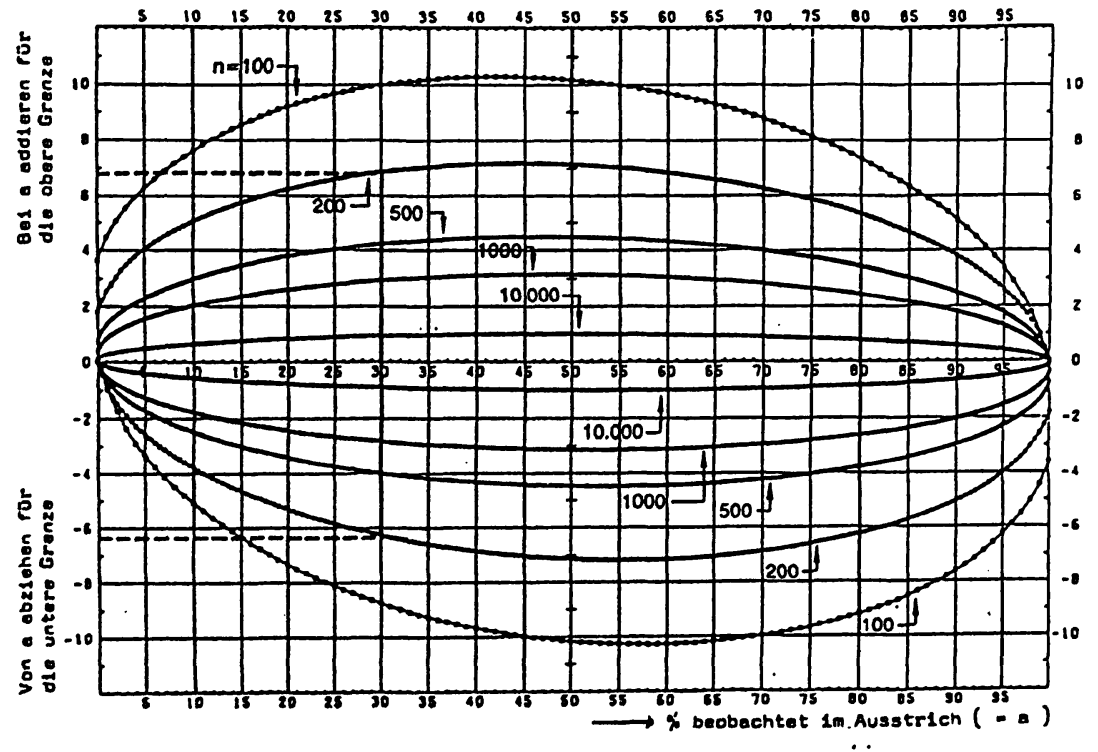

.
Abb. 1: Nomogramm des 95\%Konfidenzintervalls für den Prozentsatz einer Leukozytenpopulation, wenn im Blutausstrich a\% dieser Population differenziert wurden von der Anzahl $n$ gezählter Leukozyten (1-3). 
und von je 5 hämatologisch geschulten MTA's unseres Laboratoriums visuell differenziert $(250$ Zellen pro Differential-Blutbild). Die Proben wurden innerhalb von zwei Stunden nach Abnahme am VCS, Coulter gemessen. Sofort im Anschluß daran wurden jeweils zwei Blutbildausstriche angefertigt.

Abschnitt II: Untersucht wurden 283 Blutproben aus der Tagesroutine unseres Labors, des Zentrallabors der Universitätsklinik Münster und einer regionalen ärztlichen Laborgemeinschaf, die mindestens $8 \%$ stabkernige Granulozyten oder jüngere Vorstufen im mikroskopischen Differentialblutbild aufwiesen. Die Untersuchung des mikroskopischen Differentialblutbildes erfolgte durch zwei hämatologisch geschulte MTA's. Ausgewertet wurden jeweils 250 Zellen. Die Proben wurden späteștens nach 4 Stunden bei den externen Proben und spätestens nach 2 Stunden bei hausinternen Proben gemäß Abschnitt I verarbeitet.

Abschnitt III: Untersucht wurden 96 selektierte Blutbilder von Patienten mit gesicherten malignen Erkrankungen des blutbildenden Systems, u. a. auch Verlaufskóntrollen von Patienten bei oder nach Chemotherapie. Die Blutproben stammten zum größten Teil aus der onkologischen Ambulanz der Universitätsklinik Gießen, Prof. Dr. $H$. Pralle. Die Proben wurden durch einen Fahrdienst der Firma Coulter innerhalb von 2-3 Stunden nach $A b$ nahme ins Zentrallabor unseres Klinikums gebracht und dort wie unter Abschnitt I beschrieben weiterverarbeitet.

\section{Zusammensetzung der Kollektive}

Für alle drei Kollektive gilt: Es wurde keine Unterteilung in geschlechtsspezifische und altersspezifische Klassen vorgenommen mit einer Ausnahme: Blutproben von Kin-

Tab. 1: Cytometrisch-volumetrische Differenzierung der Leukozytenpopulationen des peripheren Blutes von 4 Patienten zur Bestimmung der Präzision in der Serie $(n=20)$ am VCS von Coulter

\begin{tabular}{|c|c|c|c|c|c|c|c|c|}
\hline Zellpopulation & $\begin{array}{l}\text { Blutb } \\
\text { (900 L }\end{array}$ & $\begin{array}{l}\text { bild } 1 \\
\text { _euk.) }\end{array}$ & $\begin{array}{l}\text { Blutb } \\
\text { (1700 }\end{array}$ & $\begin{array}{l}\text { oild } 2 \\
\text { Leuk.) }\end{array}$ & $\begin{array}{l}\text { Bluth } \\
\text { (5000 }\end{array}$ & $\begin{array}{l}\text { bild } 3 \\
\text { Leuk.) }\end{array}$ & $\begin{array}{l}\text { Blutb } \\
\text { (19600 }\end{array}$ & $\begin{array}{l}\text { bild } 4 \\
\text { Leuk. }\end{array}$ \\
\hline & & VK(\%) & $\%$ & VK(\%) & & VK $(\%)$ & $\%$ & VK(\%) \\
\hline Lymphozyten & 35,14 & 3,4 & 16,26 & 2,1 & 29,64 & 1,2 & 15,83 & 1,8 \\
\hline Monozyten & 23,76 & 11,4 & 5,15 & 7,3 & 5,55 & 8,0 & 3,26 & 4,3 \\
\hline Neutrophile & 39,27 & 2,1 & 73,93 & 1,4 & 60,37 & 0,6 & 79,93 & 0,7 \\
\hline Eosinophile & 0,84 & 7,5 & 1,32 & 8,6 & 3,31 & 6,1 & 0,85 & 6,1 \\
\hline Basophile & 1,00 & 34,3 & 3,34 & 28,6 & 1,14 & 20,1 & 0,13 & 21,1 \\
\hline
\end{tabular}

dern inter 15 Jahren wurden nicht in die Evaluation mit übernommen.

Das Kollektiv I setzt sich ausschließlich aus normal gesunden Probanden ohne Erkrankungen zusammen.

Das Kollektiv II setzt sich zu über $\mathbf{9 0 \%}$ aus Fällen mit schweren entzündlichen Erkrankungsbildern unterschiedlicher Genese zusammen. Etwa $5 \%$ der Fälle entstammen reaktiven Granulozytosen aufgrund metabolischer Erkrankungen und Schockzustände (teilweise mit Vorliegen einer toxischen Granulation (4 Blutbilder).

\section{Mikroskopische Differenzierung}

Jeder Blutausstrich erfolgte aus Gründen der Standardisierung mittels eines automatisierten Verfahrens (Spinnerausstrich; $500 \mu$ l EDTA-Blut). Nach Lufttrocknen der Präparate erfolgte die Färbung nach Pappenheim. Die mikroskopische Auswertung wuirde an zwei Leitz Dialux Mikroskopen, 100fach Objektiv NPL Fluotar 100, Ölimmersion durchgeführt. Pro Ausstrich wurden 250 Zellen differenziert (bei Proben des Abschnittes III in Sonderfällen 100 Zellen). Blutbilder des Kollektivs III wurden von einer hämatologisch/onkologisch erfahrenen MTA unșeres Labors und einer weiteren MTA (hämatologisch geschult im Zentrallabor der Universitätsklinik Münster) differenziert. Ausgesuchte Blutbilder (20 von 96) wurden zusätzlich zur Kontrolle im onkologischen Labor der Universitätsklinik Gießen, Prof. Dr. H. Pralle, differenziert.

Den jeweils Untersuchenden war das Ergebnis der mechanisierten Analyse am Coulter VCS sowie den parallel erstellten mikroskopischen Blutbildern nicht bekannt.

\section{Statistische Methoden}

Die prozentualen Verteilungen der Zellpopulationen von visueller und automatisierter Differenzierung am VCS wurden mittels linearer Regression nach Passing und Bablok (14) und durch die Ermittlung der standardisierten Hauptkomponente (15-16) statistisch ausgewertet.

\section{Flagsetzung des VCS bei pathologischen Proben}

Kriterien für das Setzen der unterschiedlichen Flags lassen sich in nachstehender Patentschrift nachlesen (17).

Tab. 2: Ermittlung der Verdünnungslinearität der Messungen von Zellpopulationen des Differentialblutbildes am VCS von Coulter (Verdünnungen -, 1:2, 1:5 und 1:10)

\begin{tabular}{|c|c|c|c|c|c|c|}
\hline Blutbild & Verdünnung & $\begin{array}{c}\text { Lymphozyten } \\
\%\end{array}$ & $\underset{\%}{\text { Monozyten }}$ & $\begin{array}{c}\text { Neutrophile } \\
\%\end{array}$ & $\begin{array}{c}\text { Eosinophile } \\
\%\end{array}$ & $\begin{array}{c}\text { Basophile } \\
\%\end{array}$ \\
\hline I (10900 Leukozyten) & $\begin{array}{l}- \\
1: 2 \\
1: 5 \\
1: 10\end{array}$ & $\begin{array}{r}10,52 \\
9,27 \\
9,57 \\
11,63\end{array}$ & $\begin{array}{l}3,97 \\
2,07 \\
2,24 \\
2,21\end{array}$ & $\begin{array}{l}85,01 \\
87,93 \\
87,67 \\
85,20\end{array}$ & $\begin{array}{l}0,36 \\
0,34 \\
0,33 \\
0,25\end{array}$ & $\begin{array}{l}0,14 \\
0,39 \\
0,19 \\
0,71\end{array}$ \\
\hline II (6700 Leukozyten) & $\begin{array}{l}- \\
1: 1 \\
1: 5 \\
1: 10\end{array}$ & $\begin{array}{r}11,41 \\
10,32 \\
9,36 \\
10,18\end{array}$ & $\begin{array}{l}6,92 \\
5,46 \\
5,85 \\
6,01\end{array}$ & $\begin{array}{l}81,15 \\
83,69 \\
84,20 \\
83,14\end{array}$ & $\begin{array}{l}0,24 \\
0,39 \\
0,36 \\
0,34\end{array}$ & $\begin{array}{l}0,28 \\
0,14 \\
0,23 \\
0,33\end{array}$ \\
\hline III (5400 Leukozyten) & $\begin{array}{l}- \\
1: 1 \\
1: 5 \\
1: 10\end{array}$ & $\begin{array}{l}28,41 \\
29,31 \\
27,43 \\
26,98\end{array}$ & $\begin{array}{l}4,67 \\
3,89 \\
5,01 \\
5,61\end{array}$ & $\begin{array}{l}64,85 \\
64,46 \\
65,93 \\
65,48\end{array}$ & $\begin{array}{l}0,84 \\
0,78 \\
0,34 \\
0,89\end{array}$ & $\begin{array}{l}1,23 \\
1,56 \\
1,29 \\
1,04\end{array}$ \\
\hline
\end{tabular}




\section{Rontrollogen,}

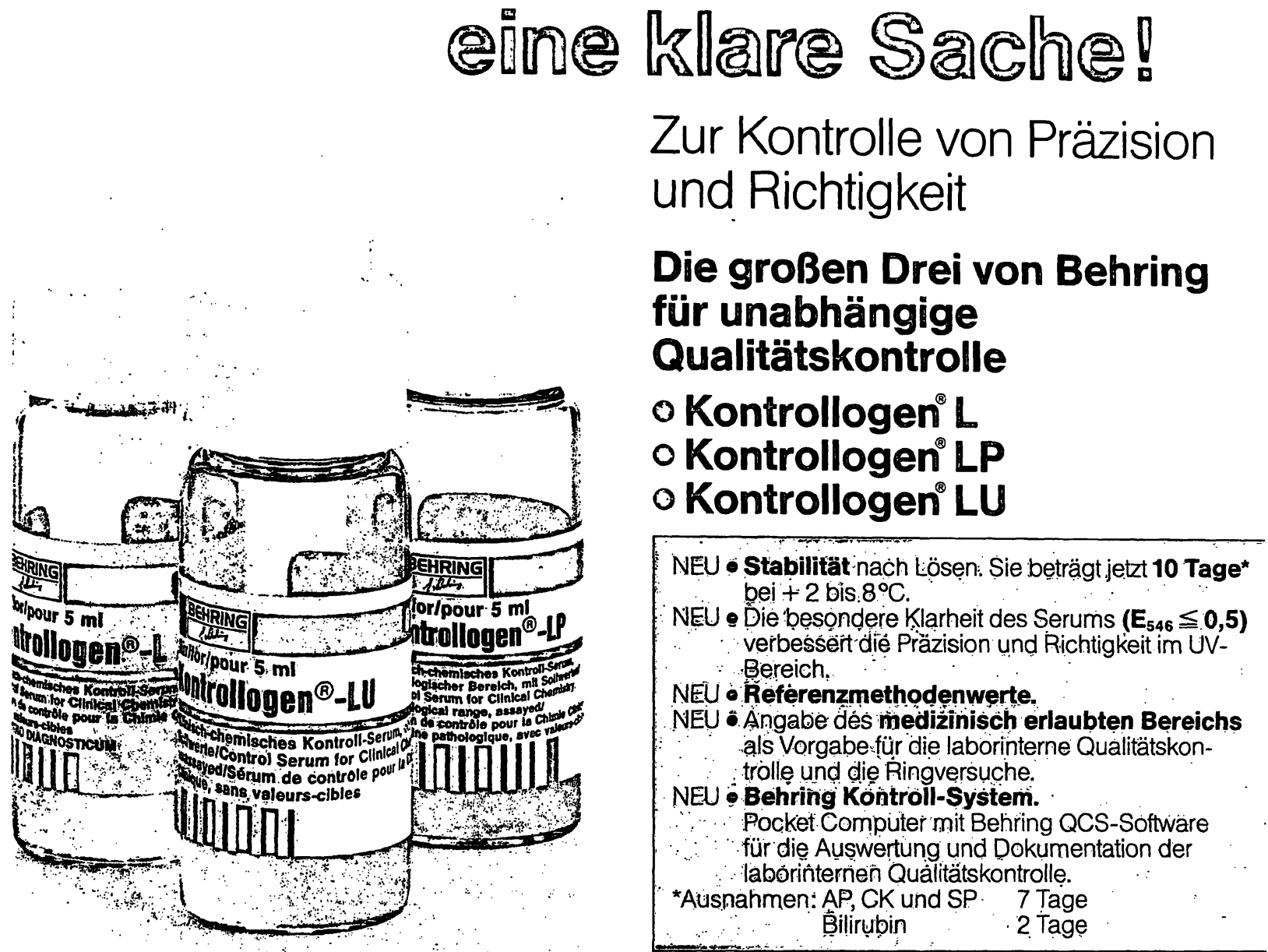

Behring. Ihr Partner für Präzision und Richtigkeit.

is Behrong Kontrollssystemeffeßt

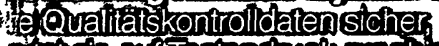
artet sieauf Tastendruck graphisch arnumerischausund dokumenttert advuerha ât

Prdernsieblitte aisfintilche Unterlagen Derdasneuekontrologen vid das Behring Kontroll=Systeman

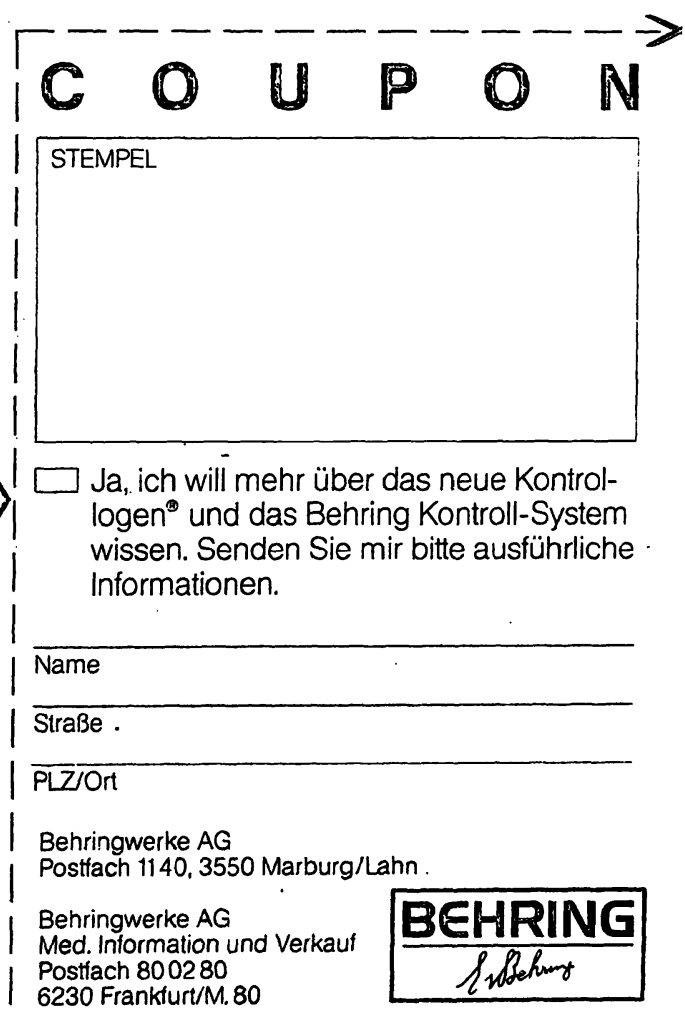




\section{Orraulitiöit.}

\section{Selbst mikroskopisch kleine Details sind für uns wichtig.}

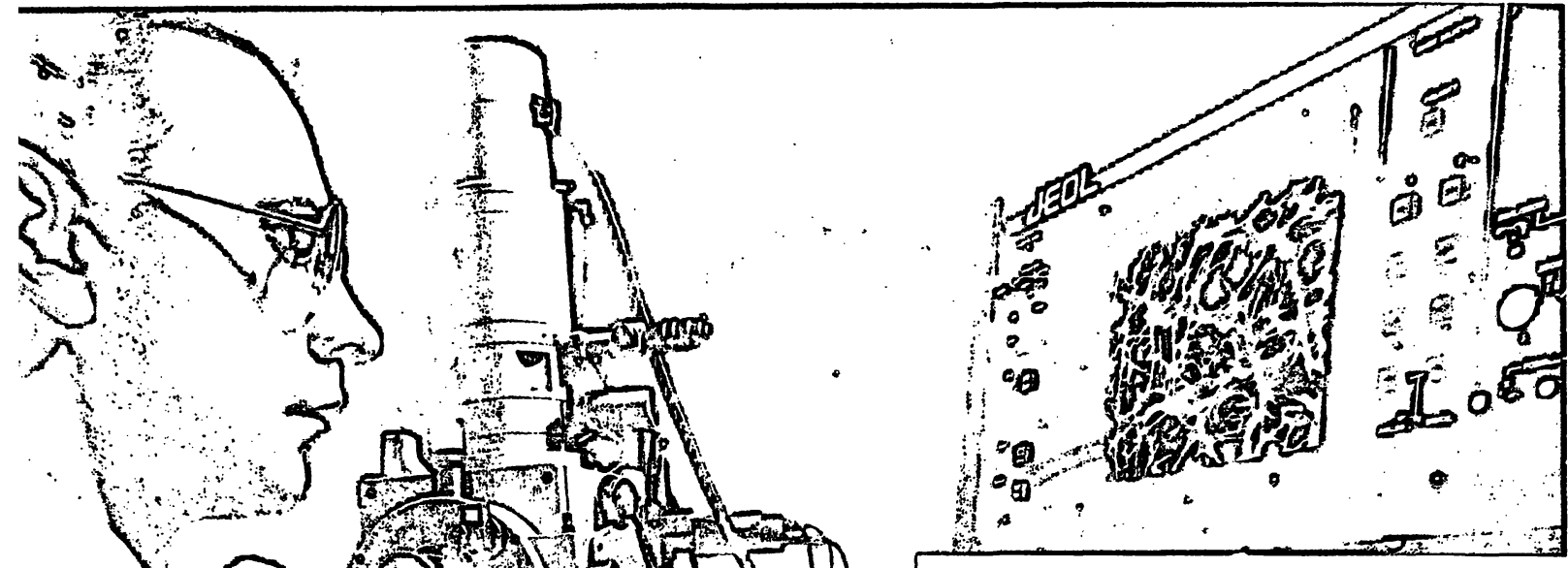

Für uns reicht die Verpflichtung für Qualität bis in die mikroskopischen Details all unserer Produkte.

So zum Beispiel bei der Entwicklung der Membrane, die in unseren Oxygenatoren und Blutfiltern verwendet werden.

Unsere Untersuchungen begannen beim Material selbst. Wir sorgten für eine wesentliche Verfeinerung der Porengröße und -form.

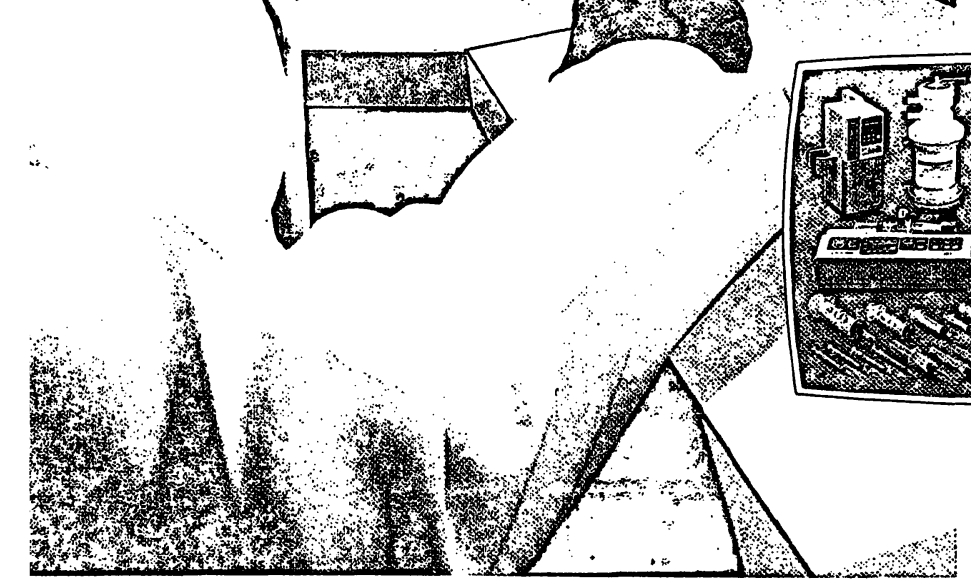

Wir überprüften Festigkeit und Leistung. Hierzu stehen uns bei Terumo Elektronenmikroskope und andere AnalyseInstrumente neuester Bauart zur Verfügung.

Es sind eben diese winzigen und doch extrem wichtigen Details, welche die "Terumo-Qualität" ausmachen. Unsere Entwicklungstechniken kommen in jedem Terumo-Erzeugnis zum Ausdruck, von der kleinsten Kanüle bis zum hochentwickelten künstlichen Organ. Bei Terumo achten wir auf Qualität bis ins letzte Detail. Das bedeutet für Sie höchste Sicherheit und Zuverlässigkeit des Erzeugnisses. Dazu steht Terumo.
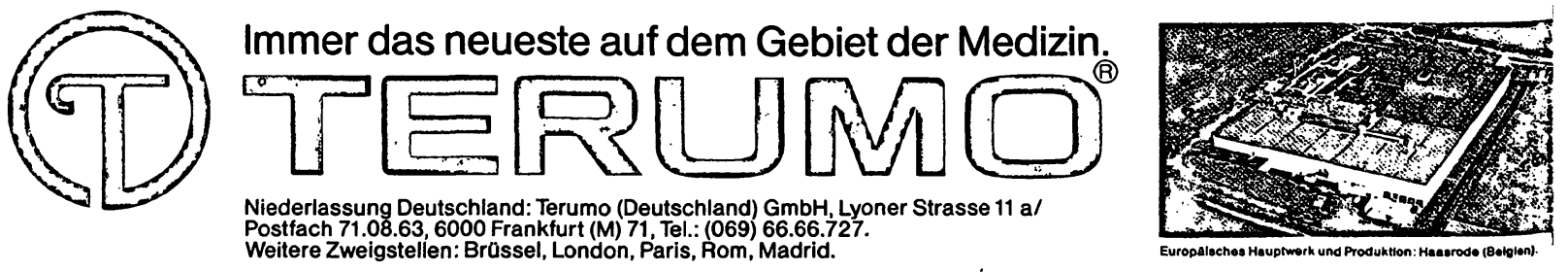


\section{Ergebnisse}

Präzision in der Serie $(n=20)$

Die Präzision der Differentialblutbild-Messung wurde anhand von 4 Blutbildern mit 900, 1700, 5000 und 19600 Gesamt-Leukozytenzahl ermittelt. Jedes Blutbild wurde $20 \mathrm{mal}$ am VCS von Coulter gemessen und der jeweilige VK der Zellpopulation ermittelt (s. Tab. 1). Vergleicht man diese Ergebnisse mit z. B. von Thomas et al. (8) durchgeführten Ergebnissen zur visuellen Mikroskopie (Auszählen eines Blutbildes an 10 verschiedenen Tagen zu je 500 Zellen) (VK zwischen 10,1 und $48 \%$ ), so sind die Präzi-' sionswerte am VCS zwar abhängig von der Gesamtleukozytenzahl, aber insgesamt wesentlich besser als bei der visuellen Mikroskopie.

\section{Linearität der Messung (Verdünnungslinearität)}

Es wurden an drei Blutbildern Verdünnungsexperimente mit homologem EDTA-Plasma durchgeführt (Tab. 2). Die Messungen mit $1: 1,1: 5$ und $1: 10$ Verdünnungen zeigen keine Abweichung von der Linearität.

\section{Verschleppungseffekte}

Versuche zur Fragestellung von Verschleppungseffekten wurden mit drei Proben durchgeführt, die alternierend gemessen wurden:
Probe 1: Monomyelozytäre Leukämie (Leukozyten 98000 ) Probe 2: Normales Blutbild (Leukozyten 5000 )

Probe 3: Chemotherapiertes Mammakarzinom (Leukozyten 400)

Nennenswerte Verschleppungseffekte waren nur bei alternierender Messung von Probe 1 und Probe 3 feststellbar: Probe 3 vor Verschleppungsversuch (Mittelwerte $n=5$ ): Lymphozyten $74,91 \%$, neutrophile Granulozyten $6,06 \%$, Monozyten $17,03 \%$, eosinophile Granulozyten $1,34 \%$, basophile Granulozyten $0,65 \%$. Probe 3 nach Verschleppungsversuch mit Probe 1 (Mittelwert $n=5$ ): Lymphozyten $63,81 \%$, neutrophile Granulozyten $11,29 \%$, Monozyten $22,71 \%$, eosinophile Granulozyten $1,18 \%$, basophile Granulozyten 1,01\%.

Vergleich der Differenzierungsergebnisse VCS versus visuelle Mikroskopie - Untersuchungsergebnisse bei normalen Blutbildern

Die nach Passing und Bablok durchgeführte lineare Regression (statistische Auswertung eines Methodenvergleichs, bei dem beide Methoden mit Fehlern behaftet sind) ergab für neutrophile Granulozyten ( $r=0,952)$ (Abb. 2, Tab. 3), eosinophile Granulozyten ( $r=0,96)$ (Abb. 3, Tab: 3), Lymphozyten ( $r=0,952$ ) (Abb. 4, Tab. 3) eine gute Korrelation, sowie bei den Monozyten $(r=$ 0,693 ) (Abb. 5, Tab. 3) eine nur mäßige Korrelation. Keine

Tab. 3: Methodenvergleich an Patientenproben des Kollektivs I (normale Blutbilder) zwischen mikroskopischer $(X)$ und Differenzierung am VCS $(Y) Y=a+b(X), N=96$. Die Konfidenzintervalle von a und b nach Passing/Bablok sind in Klammern gesetzt; ma 68, ma 95 - Streuung der Residuen um Passing/Bablok, $S_{x / y}=$ Standardfehler der Residuen um die standardisierte Hauptkomponente, $r=K o r r e-$ lationskoeffizient

\begin{tabular}{|c|c|c|c|c|c|c|c|c|}
\hline \multirow[t]{2}{*}{ Darstellung } & \multicolumn{4}{|c|}{ Passing/Bablok } & \multicolumn{3}{|c|}{ Stand. Hauptkomponente } & \multirow[t]{2}{*}{$\mathbf{r}$} \\
\hline & $\mathbf{a}$ & $\mathbf{b}$ & ma 68 & $\operatorname{ma} 95$ & $\mathbf{a}$ & b & $S_{x / y}$ & \\
\hline Lymphozyten & $\begin{array}{l}-2,97 \\
(-4,749- \\
-1,335)\end{array}$ & $\begin{array}{c}0,961 \\
(0,898- \\
1,018)\end{array}$ & 2,051 & 4,823 & $-3,622$ & 0,959 & 2,225 & 0,952 \\
\hline $\begin{array}{l}\text { Neutrophile } \\
\text { Granulozyten }\end{array}$ & $\begin{array}{c}0,215 \\
(-3,009- \\
3,217)\end{array}$ & $\begin{array}{c}1,020 \\
(0,971- \\
1,075)\end{array}$ & 1,975 & 6,502 & 1,013 & 1,023 & 2,532 & 0,952 \\
\hline $\begin{array}{l}\text { Eosinophile } \\
\text { Granulozyten }\end{array}$ & $\begin{array}{c}0,001 \\
(-0,247- \\
0,149)\end{array}$ & $\begin{array}{c}1,293 \\
(1,182- \\
1,434)\end{array}$ & 0,423 & 1,757 & $\quad 0,222$ & 1,240 & 0,758 & 0,96 \\
\hline Monozyten & $\begin{array}{c}-2,384 \\
(-4,672- \\
-0,902)\end{array}$ & $\begin{array}{c}1,714 \\
(1,457- \\
2,067)\end{array}$ & 1,151 & 2,056 & $-2,016$ & 1,573 & 1,464 & 0,693 \\
\hline
\end{tabular}

Tab. 4: Methodenvergleich an Patientenproben des Kollektivs II (reaktiv-linksverschobene Blutbilder) zwischen mikroskopischer (X) und Differenzierung am VCS $(Y) Y=a+b(X), N=283$. Die Konfidenzintervalle von a und $b$ nach Passing/Bablok sind in Klammern gesetzt; ma 68, ma 95 - Streuung der Residuen um Passing/Bablok, $S_{x y}=$ Standardfehler der Residuen um die standardisierte Hauptkomponente, $r=$ Korrelationskoeffizient

\begin{tabular}{|c|c|c|c|c|c|c|c|c|}
\hline \multirow[t]{2}{*}{ Darstellung } & \multicolumn{4}{|c|}{ Passing/Bablok } & \multicolumn{3}{|c|}{ Stand. Hauptkomponente } & \multirow[t]{2}{*}{$\mathbf{r}$} \\
\hline & a & b & ma 68 & ma 95 & a & b - & $S_{x / y}$ & \\
\hline Lymphozyten & $\begin{array}{l}-1,019 \\
(-1,531- \\
-0,676)\end{array}$ & $\begin{array}{c}0,91 \\
(0,867- \\
0,972)\end{array}$ & 1,728 & 5,915 & $-2,424$ & 1,029 & 2,990 & 0,941 \\
\hline $\begin{array}{l}\text { Neutrophile } \\
\text { Granulozyten }\end{array}$ & $\begin{array}{c}1,321 \\
(-1,217- \\
4,350)\end{array}$ & $\begin{array}{c}0,994 \\
(0,958- \\
1,027)\end{array}$ & 2,005 & 5,696 & $-2,257$ & 1,039 & 4,259 & 0,924 \\
\hline $\begin{array}{l}\text { Eosinophile } \\
\text { Granulozyten }\end{array}$ & $\begin{array}{c}0,144 \\
(-0,12- \\
0,168)\end{array}$ & $\begin{array}{c}0,979 \\
(0,922- \\
1,05)\end{array}$ & 0,285 & 1,296 & 0,118 & 1,005 & 0,541 & 0,941 \\
\hline Monozyten & $\begin{array}{c}0,146 \\
(-0,335- \\
0,557)\end{array}$ & $\begin{array}{c}1,120 \\
(1,018- \\
1,224)\end{array}$ & 1,483 & 4,856 & $-2,154$ & 1,468 & $\begin{array}{c}5,307 \\
.\end{array}$ & 0,510 \\
\hline
\end{tabular}


Korrelation ergab sich für die basophilen Granulozyten (Abb. 6). Der Cusum-Test auf Abweichungen von der Linearität für basophile Granulozyten war statistisch signifikant, so daß eine Auswertung nach Passing/Bablok nicht möglich ist.

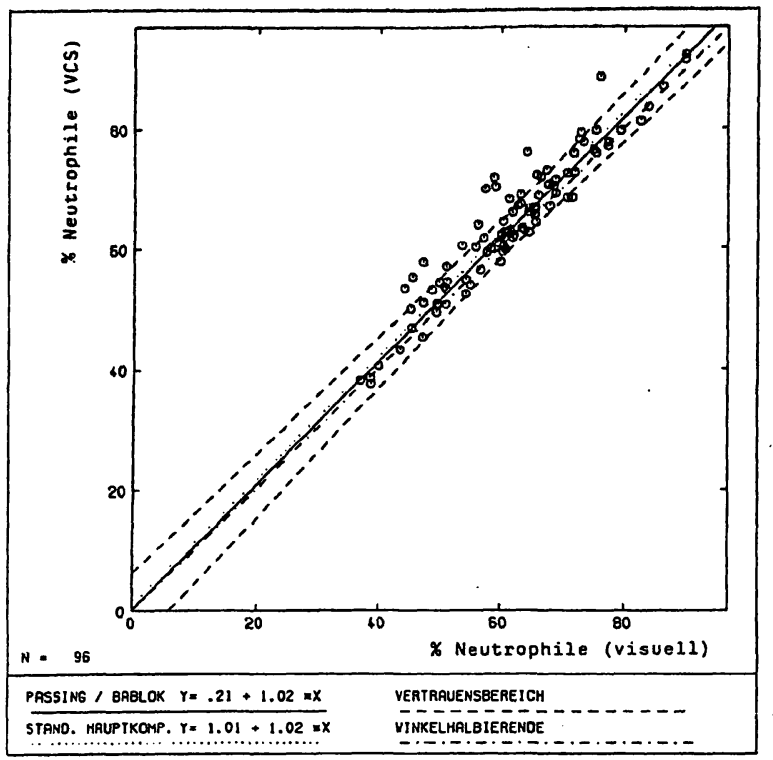

Abb. 2: Vergleich der neutrophilen Granulozyten (Prozent) des Kollektivs normale Blutbilder zwischen visueller Differenzierung $(X)$ und Bestimmung am VCS von Coulter (Y). Auswertung nach Passing/Bablok und Angabe der standardisierten Hauptkomponente des Methodenvergleichs (s. Tab. 1).

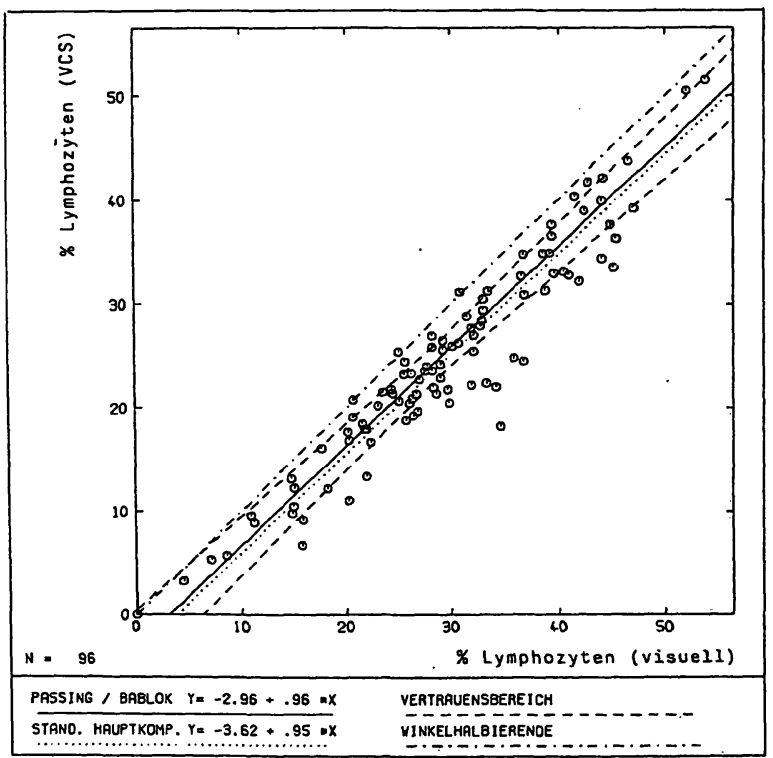

Abb. 4: Vergleich der Lymphozyten (Prozent) von Patientenproben des Kollektivs normale Blutbilder zwischen visueller Differenzierung $(X)$ und Bestimmung am VCS von Coulter $(Y)$. Auswertung nach Passing/Bablok und Angabe der standardisierten Hauptkomponente des Methodenvergleichs (s. Tab. 1).
Das Gerät VCS zeigte eine sehr gute Übereinstimmung zum visuellen Differentialblutbild im Fall der neutrophilen Granulozyten $(Y=1,02 X+0,215)$, ferner eine leichte proportionale Erhöhung bei den eosinophilen Granulozyten $(Y=1,293 X+0,001)$ sowie eine Verminderung der

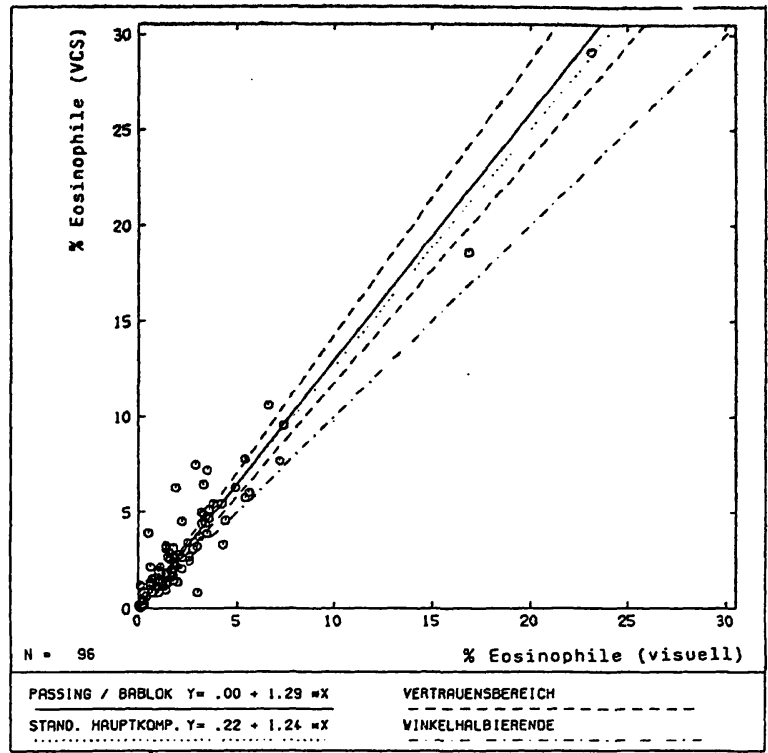

Abb. 3: Vergleich der eosinophilen Granulozyten (Prozent) des Kollektivs normale Blutbilder zwischen visueller Differenzierung $(X)$ und Bestimmung am VCS von Coulter $(Y)$. Auswertung nach Passing/Bablok und Angabe der standardisierten Hauptkomponente des Methodenvergleichs (s. Tab. 1).

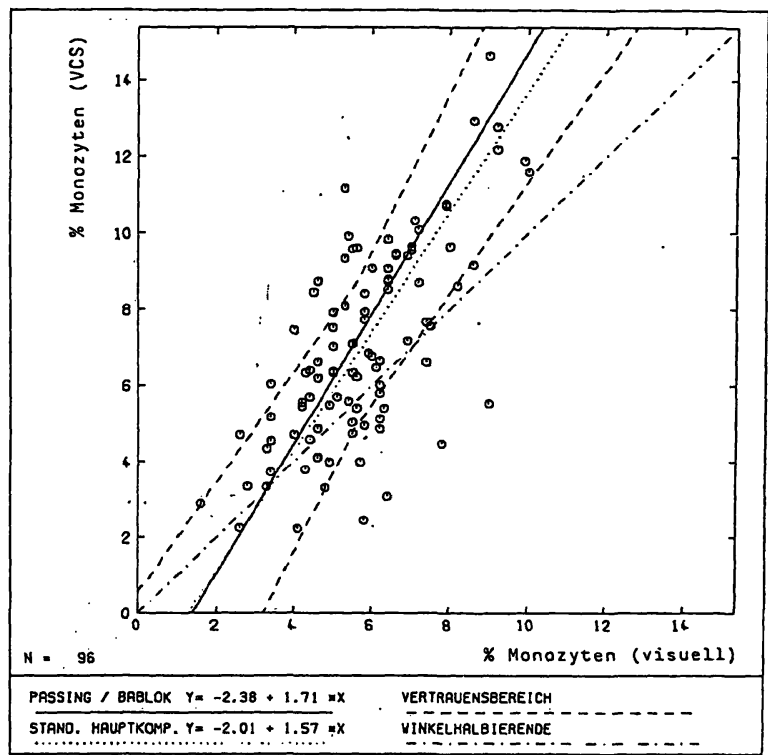

Abb. 5: Vergleich der Monozyten (Prozent) von Patientenproben des Kollektivs normale Blutbilder zwischen visueller Differenzierung $(X)$ f und Bestimmung am VCS von Coulter (Y). Auswertung nach Passing/Bablok und Angabe der standardisierten Hauptkomponente des Methodenverg/eichs (s. Tab. 1). 
Lymphozytenzahlen im relevanten Bereich um 10-20\% $(Y=0,961 X-2,97)$.

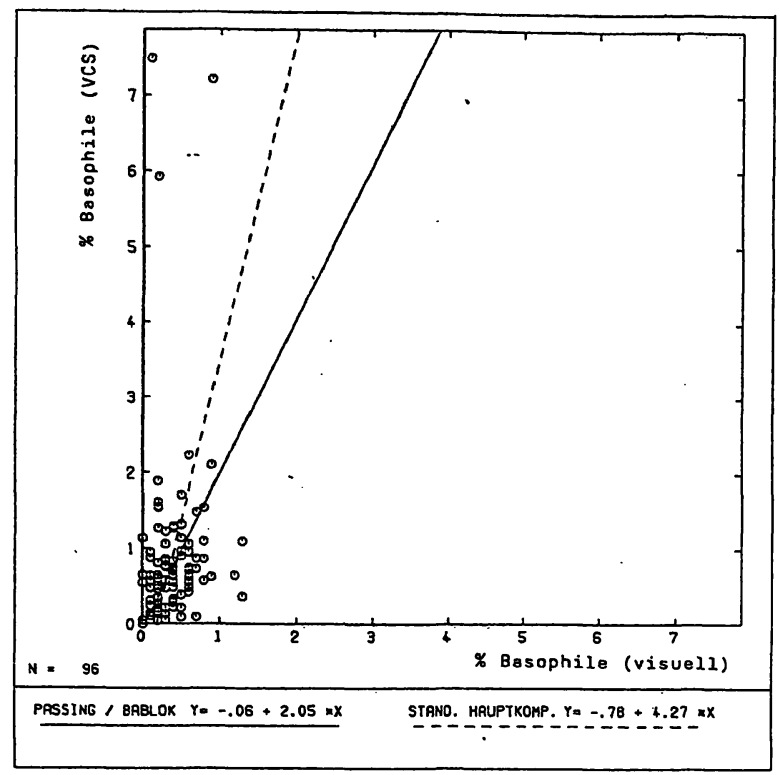

Abb. 6: Vergleich der basophilen Granolozyten (Prozent) des Kollektivs normale Blutbilder zwischen visueller Differenzierung $(X)$ und Bestimmung am VCS von Coulter (Y). Auswertung nach Passing/Bablok und Angabe der standardisierten Hauptkomponente des Methodenvergleichs sind nicht zulässig, da der Cusum-Test durch seine Signifikanz eine Abweichung von der Linearität anzeigt.

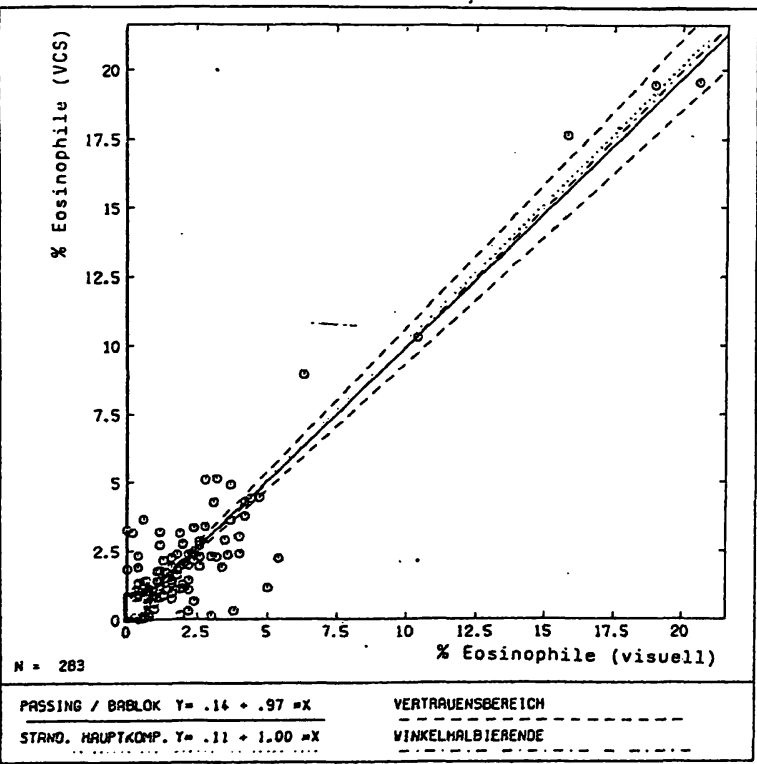

Abb. 8: Vergleich der eosinophilen Granulozyten (Prozent) von Patientenproben des Kollektivs II (reaktiv-linksverschobene Blutbilder) zwischen visueller Differenzierung $(X)$ und Bestimmung am VCS von Coulter (Y). Auswertung nach Passing/Bablok und Angabe der standardisierten Hauptkomponente des Methoden: vergleichs (s. Tab. 2).
Untersuchungsergebnisse bei linksverschobenen Blutbildern

Die. nach Passing und Bablok durchgeführte lineare Regression ergab für neutrophile Granulozyten $(Y=0,994 X$

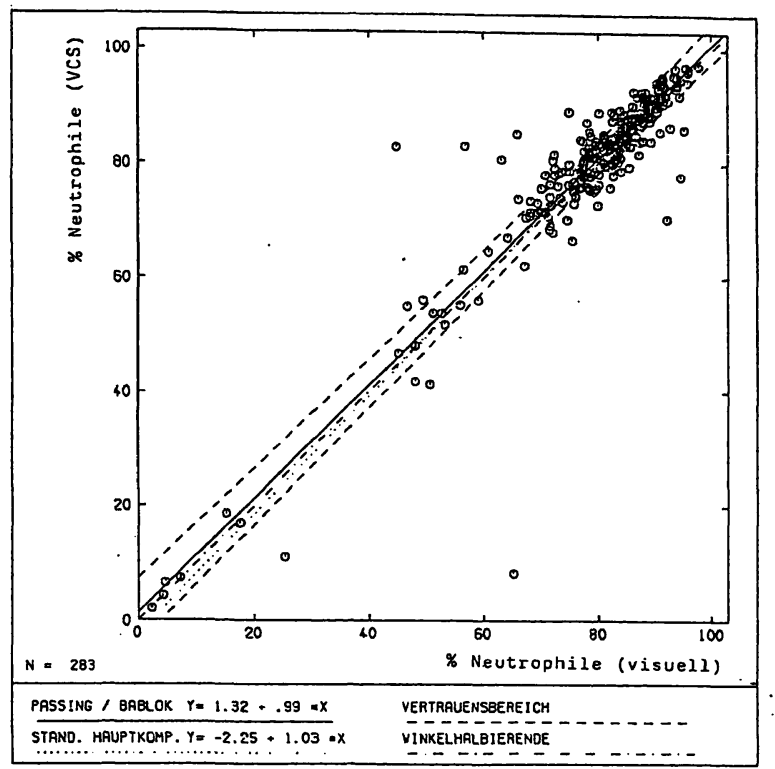

Abb. 7: Vergleich der neutrophilen Granulozyten (Prozent) von Patientenproben des Kollektivs II (reaktiv-linksverschobene Blutbilder) zwischen visueller Differenzierung $(X)$ und Bestimmung am VCS von Coulter (Y). Auswertung nach Passing/Bablok und Angabe der standardisierten Hauptkomponente des Methodenvergleichs (s. Tab. 2).

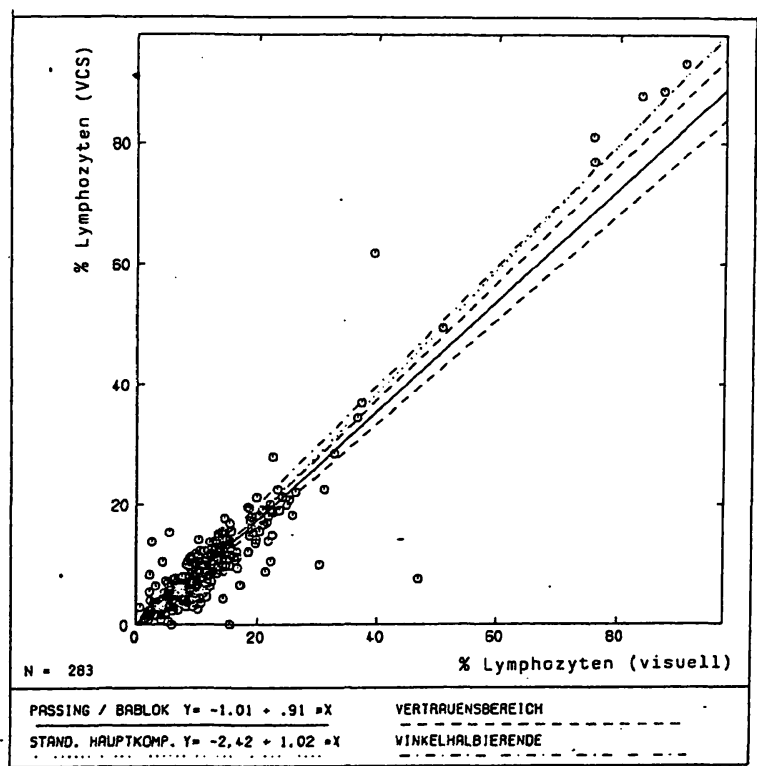

Abb. 9: Vergleich der Lymphozyten (Prozent) von Patientenproben des Kollektivs II (reaktiv-linksverschobene Blutbilder) zwischen visueller Differenzierung $(X)$ und Bestimmung am VCS von Coulter (Y). Auswertung nach Passing/Bablok und Angabe der standardisierten Hauptkomponente des Methodenvergleichs (s. Tab. 2). 
+ 1,321, $r=0,924$ ) (Abb. 7, Tab. 4), eosinophile Granulozyten $(Y=0,979 X+0,144, r=0,941)$ (Abb. 8, Tab. 4) und Lymphozyten ( $Y=0,91 X-1,019, r=0,941$ ) (Abb. 9, Tab. 4) eine gute Korrelation, sowie bei Monozyten ( $Y=1,12 X$ $+0,146, r=0,51$ ) (Abb. 10, Tab. 4) eine nur mäßige Korrelation. Wiederum zeigte der Cusum-Test bei den basophiIen Granulozyten eine Abweichung von der Linearität an. Auch in diesem Fall waren die basophilen Granulozyten nicht nach Passing/Bablok auszuwerten (Abb. 11).

Effizienzbetrachtung für den VCS von Coulter in der Erkennung von reaktiv-linksverschobenen Differentialblutbildern

Die Beurteilung der Sensitivität erfolgte auf der Grundlage der Anzeige von sogenannten "X-Flags" des VCS. Geräte-interne Spezifikationen des VCS für die Kategorie abnormal wurden nicht berücksichtigt.

Bei Betrachtung des 283 Proben umfassenden Kollektivs von reaktiv-linksverschobenen Blutbildern konnte eine Falsch-positiv-Rate von $0 \%$ und eine Falsch-negativ-Rate von $1,04 \%$ ermittelt werden. Somit lag die Effizienz des VCS von Coulter für die Erkennung von reaktiv-linksverschobenen Differentialblutbildern bei $98,96 \%$ (Tab. 5).

Untersuchungsergebnisse bei pathologischen Blutbildern von Patienten mit malignen Erkrankungen des blutbildenden Systems

Von 96 im mikroskopischen Präparat als pathologisch eingestuften Blutbildern wurden 77 vom VCS-System als pathologisch durch "X-Flags" korrekt indiziert. Die restlichen 19 Blutbilder wurden vom VCS als falsch negativ klassifiziert $(19,6 \%)$. Nur vier der vom VCS als falsch negativ klassifizierten Blutbilder wiesen pathologische Leukozytenzahlen $<1300 / \mu l$ auf. Die Effizienz des VCS von Coulter für die Erkennung hochpathologischer Differential-Blutbilder lag demnach bei $80,2 \%$ (unter Berücksichtigung der absoluten Leukozytenzahlen bei $84,4 \%$ )

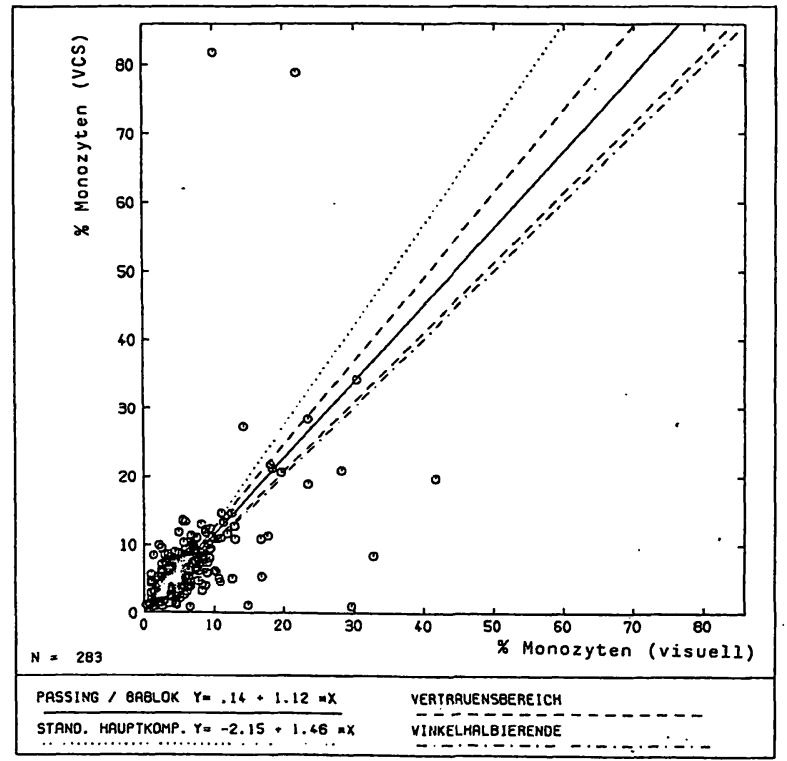

Abb. 10: Vergleich der Monozyten (Prozent) von Patientenproben des Kollektivs II (reaktiv-linksverschobene Blutbilder) zwischen visueller Differenzierung $(X)$ und Bestimmung am VCS von Coulter (Y). Auswertung nach Passing/Bablok und Angabe der standardisierten Hauptkomponente des Methodenvergleichs (s. Tab. 2).
Tab. 5: Daten zur Sensitivität und Spezifität des Coulter vCS in der Erkennung reaktiv-linksverschobener Blutbilder

\begin{tabular}{lcc}
\hline & VCS pathologisch & VCS normal \\
\hline visuell pathologisch & 283 & 3 \\
visuell normal & 0 & 0 \\
\hline
\end{tabular}

Tab. 6: Daten zur Sensitivität und Spezifität des Coulter VCS in der Erkennung pathologisch-onkologischer Blutbilder

\begin{tabular}{lcc}
\hline & VCS pathologisch & VCS normal \\
\hline visuell pathologisch & $7(81)$ & $19(15)$ \\
visuell normal & 0 & 0 \\
\hline
\end{tabular}

Werte in () unter Berücksichtigung der Leukozytenzahl

(Tab. 6). Tabelle 7 zeigt eine Auflistung des für die 19 falsch negativen Proben verfügbaren Datenmaterials. Bei 3 der 19 Blutbilder wurden nur 100 Zellen visuell differenziert (s. Asterix Tab. 7); zur Ermittlung der prozentualen Abweichung (man. Diff. - VCS Diff.) und des prozentualen Bias (in Klammern, s. Tab. 7) wurde für das Ergebnis der manuellen Differenzierung eine Mittelung der beiden parallel erstellten Ergebnisse zugrunde gelegt.

\section{Diskussion}

Seit der Einführung von Geräten zur Erstellung von automatisierten Blutbildern (Erfassung von Erythrozyten, Leukozyten, Thrombozyten, Hämoglobin, Hämatokrit und Ermittelung der Rechengrößen $\mathrm{MCV}, \mathrm{MCH}$ ) hat es nicht an Bemühungen gefehlt, auch das zeit- und personalaufwendige Differentialblutbild zu automatisieren.

Pattern recognition Systeme verfolgen als Lösungskonzept eine weitestgehende Annäherung an das visuelle

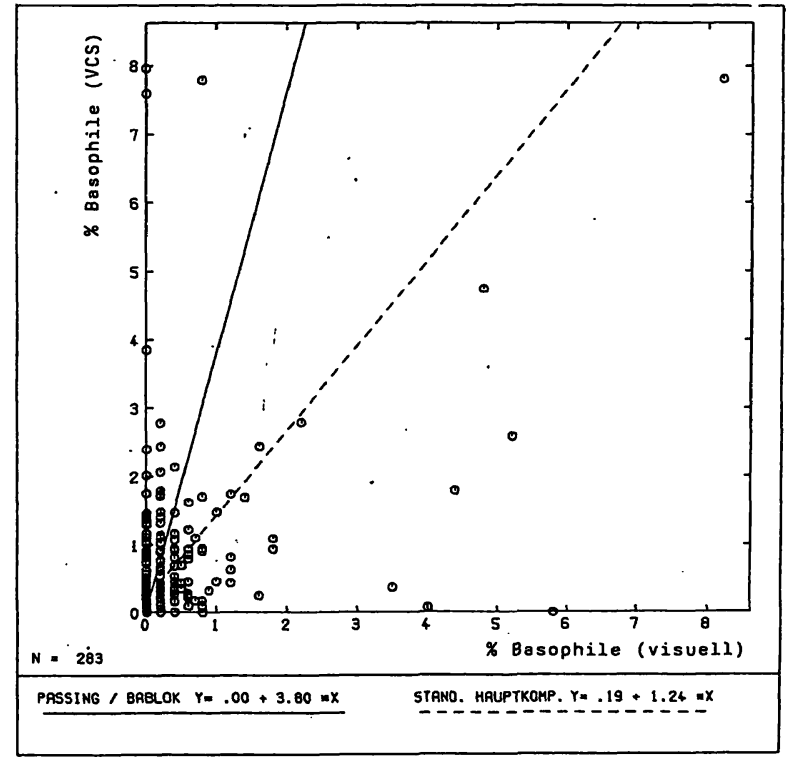

Abb. 11: Vergleich der basophilen Granulozyten (Prozent) von Patientenproben des Kollektivs II (reaktiv-linksverschobene Blutbilder) zwischen visueller Differenzierung $(X)$ und Bestimmung am VCS von Coulter (Y). Auswertung nach Passing/Bablok und Angabe der standardisierten Hauptkomponente sind nicht zulässig, da der Cusum-Test durch seine Signifikanz eine Abweichung von der Linearität anzeigt. 


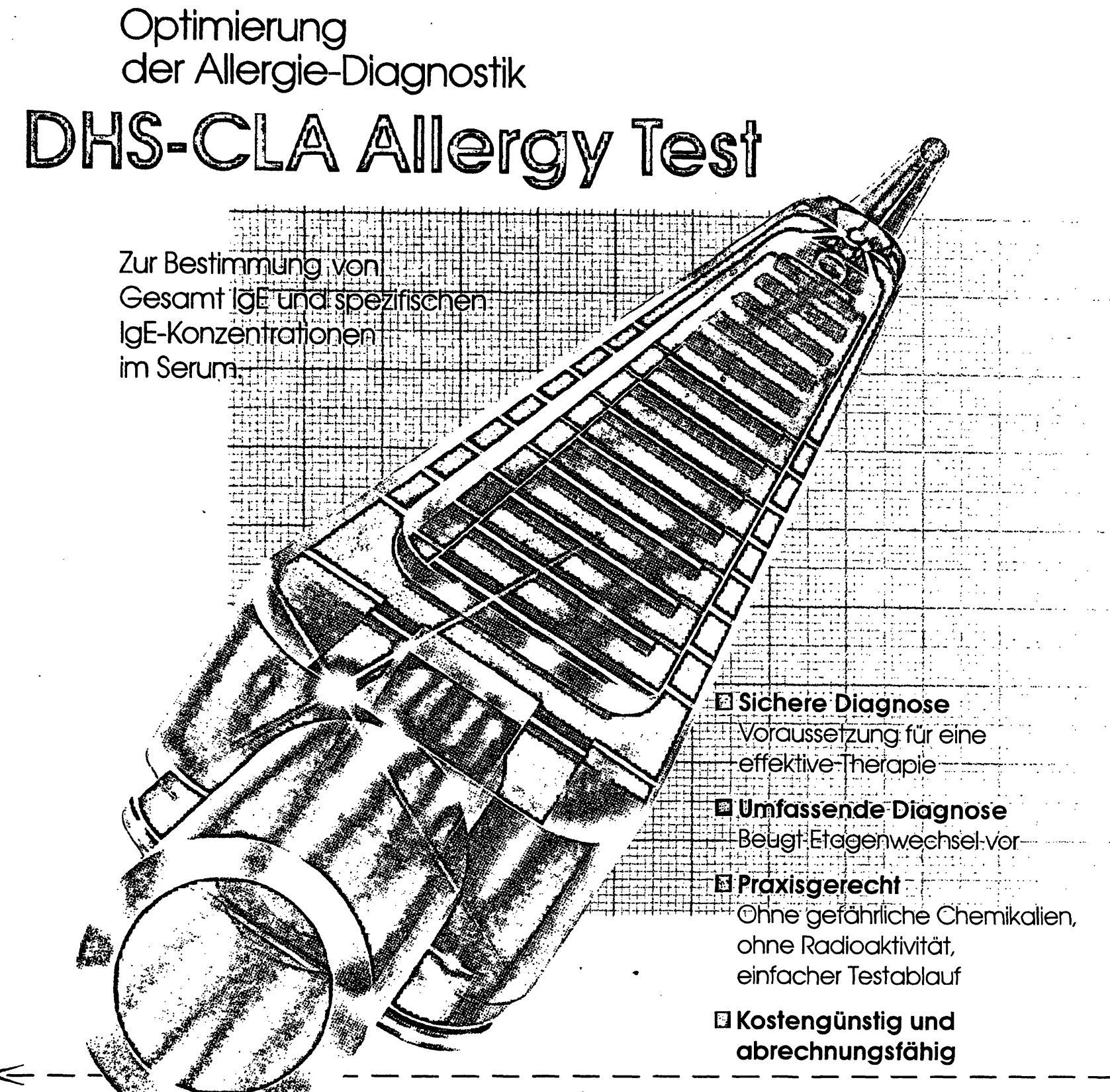

Bitte um

Z Zusendung von Information und Preisliste

Telefonisches Beratungsgespräch

$\square$ Besuch des Außendienstmitarbeiters mit Präsentation des DHS-CLA Allergy Tests

DHS/Tropon

Troponwerke

Berliner Str. 156

5000 Köln 80 


\section{IDITIION

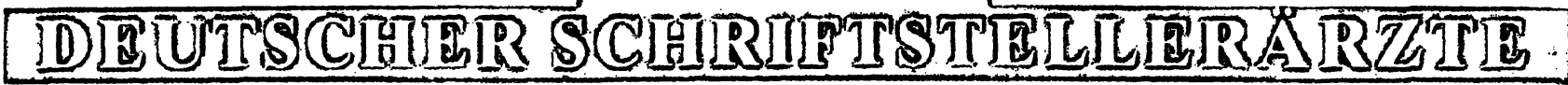

\section{Literaturpreis 1988 der Bundesärztekammer}

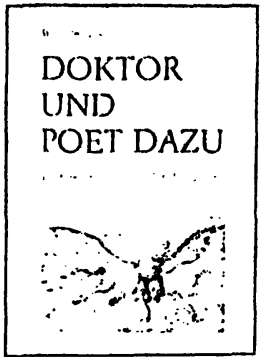

WILHELM THEOPOLD

\section{Doktor und Poet dazu}

\section{Dichterärzte}

aus sechs

Jahrhunderten

428 Seiten, geb., 39,80 DM, 2. Aufl. ISBN 3-87409-032-9

\section{KERLAC HHEIM}

Postfach 2524, 6500 Mainz

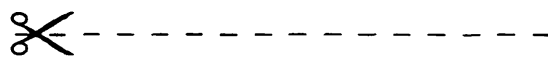

\section{Bestellschein}

Ich bestelle folgende Titel (bitte ankreuzen): $\square$ Doktor und Poet dazu $39,80 \mathrm{DM}$

D. Wenn Zaunpfähle nicht so schwer wären $12,80 \mathrm{DM}$

CDer Schwimmgürtel

$15,80 \mathrm{DM}$

$\square$ Hinter Bremsspur und Blaulicht

․ Trotz Rollstuhl und Sozialdienst

$\square$ C'est la vie

$\square$ Altred Döblin

$\square$ Sterne über fernen Ländern

$\square$ Die Porzellanpendüle

$\square$ Der Schneemann

$\square$ vorwiegend heiter

$16,80 \mathrm{DM}$

$16,80 \mathrm{DM}$

$19,80 \mathrm{DM}$

$18,00 \mathrm{DM}$

$24,80 \mathrm{DM}$

$29,80 \mathrm{DM}$

$19,80 \mathrm{DM}$

$19,80 \mathrm{DM}$

Name

Straße

PLZ

Ort

Datum/Unterschritt

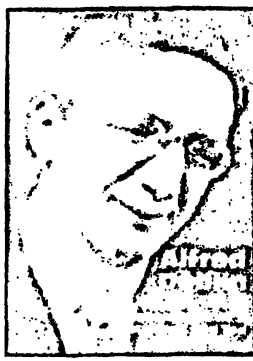

HARALD NEUMANN

Alfred Döblin

Leben und Werk

Krankheit und Tod

112 Seiten, Broschiur,

$18,00 \mathrm{DM}$

ISBN 3-87409.031-0
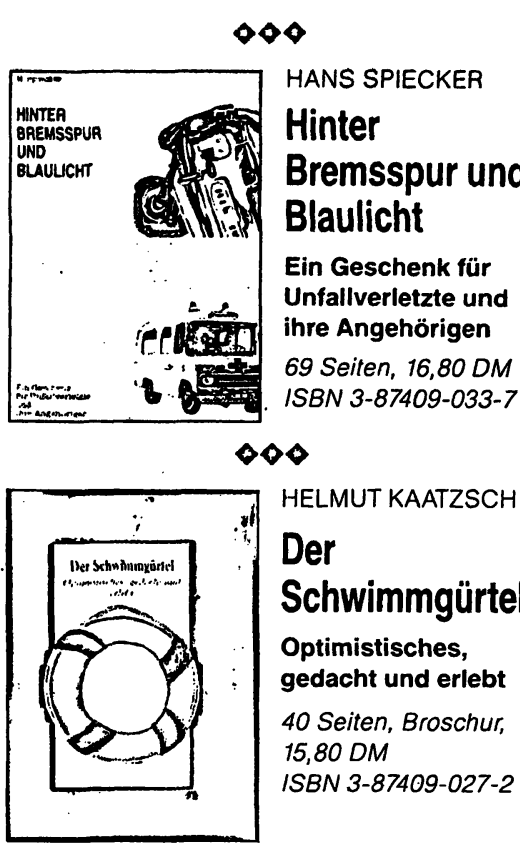

HELMUT KAATZSCH

\section{Der}

Schwimmgürtel

Optimistisches, gedacht und erlebt

40 Seiten, Broschur,

$15,80 D M$

ISBN 3-87409-027-2
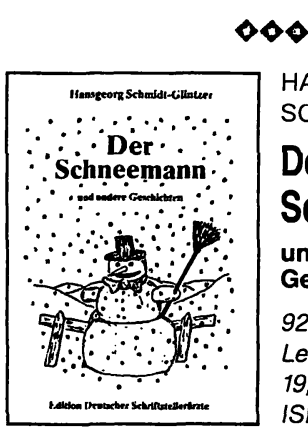

HANSGEORG

SCHMIDT-GLINTZER

Der

\section{Schneemann}

und andere

92 Seiten.

Leineneinband,

19,80 DM

ISBN 3-87409:-037-X

$\diamond \diamond \diamond$

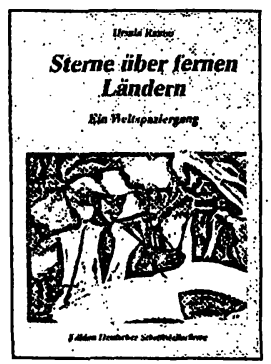

Sterne über fernen Ländern Ein Weltspaziergang 148 Seiten, Leineneinband, 24,80 DM. ISBN 3-87409-035-3
Geschichten

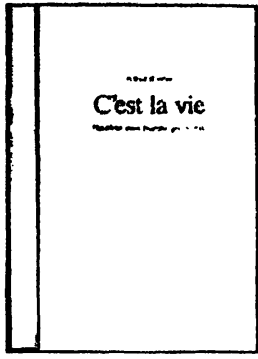

ALFRED ROTTLER

C'est la vie

Nachlese eines Nürnberger Arztes

108 Seiten, geb.,

$19,80 \mathrm{DM}$

ISBN 3-87409-034-5
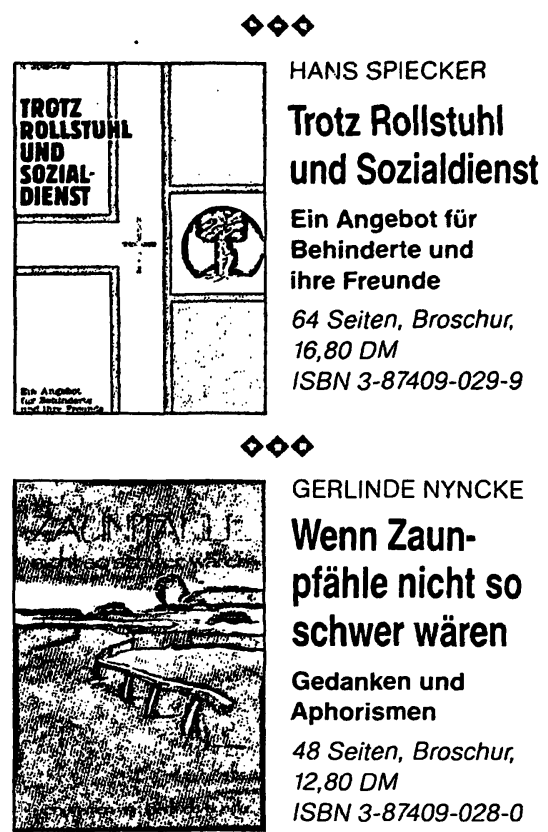

GERLINDE NYNCKE Wenn Zaunpfähle nicht so schwer wären

Gedanken und Aphorismen

48 Seiten, Broschur, $12,80 D M$

ISBN 3-87409-028-0

$\diamond \diamond$

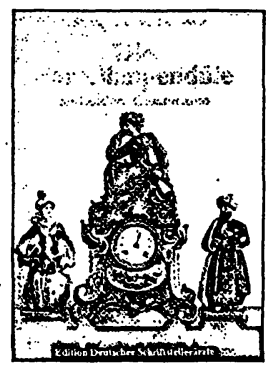

WOLFGANG LOHMANN

Die Porzellanpendüle und andere Geschichten 224 Seiten, Leineneinband, 29,80 DM ISBN 3-87409-036-1

\section{$\infty \phi$}

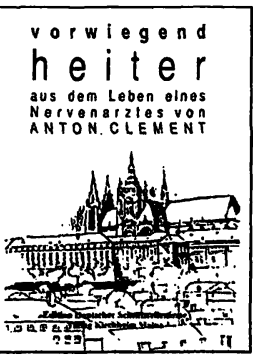

ANTON CLEMENT

\section{vorwiegend heiter \\ aus dem Leben eines Nervenarztes 88 Seiten, Leineneinband, $19,80 \mathrm{DM}$ ISBN 3-87409-039-6}


Differenzierungsverfahren. Eine Zelle wird mikroskopisch erfaßt, in ein Raster aus Tausenden von Bildprodukten zerlegt und das Bild mit eingespeichertem Datenmaterial im sehr aufwendigen Rechner verglichen. Stimmt das Zellbild mit dem "pattern" überein, so kann die Zelle klassifiziert werden. Geräte dieser Art sind so gut wie der investierte Programmieraufwand und nur dann hinreichend schnell, wenn ein extrem schneller Rechner mit dem System gekoppelt ist. Probleme ergeben sich bei der Standardisierung der Zellfärbung und durch die Tatsache der statistischen Ungenauìgkeit bei Zählung von nur 100 Zellen pro Ausstrich (1-3). Derzeit wird nur noch. ein System, welches die oben genannten Anforderungen erfüllt, auf dem europäischen Markt angeboten (Omron, Europe GmbH, Hamburg).

\section{Cytochemisch-cytometrische Verfahren}

Geräte wie das Technicon $\mathrm{H} 6000$ und Technicon $\mathrm{H} 1$ kombinieren die Peroxidase-Anfärbbarkeit der Zelle (cytoche- misch) mit der Streulichtmessung an Leukozyten (Größe der Zelle) und cytometrischer Streulichtmessung an Leukozytenkernen (Informationen über Basophile, Aussagen über reaktive/pathologische Linksverschiebung aus dem Maß der Kernsegmentierung). Diese drei Größen werden in einem zweidimensionalen Nukleogramm zu bestimmten Zellen des weißen Blutbildes empirisch korreliert. Erkennung der einzelnen Zellpopulationen, das Setzen der Diskriminationsgrenzen und das Setzen von „Flags" für pathologische Ergebnisse hängen vom Rechneraufwand und von der Gerätejustierung ab. Vorteil dieses Verfahrens ist eine erheblich höhere statistische Genauigkeit der Zellzählung (10000 Zellen).

\section{Cytometrisch-volumetrische Analysensysteme}

Geräte wie das VCS von Coulter $(8,11,12)$ analysieren und klassifizieren die Zelle des weißen Blutbildes nach folgenden Kriterien: Individuelles Zellvolumen, der Hochfrequenzleitfähigkeit als Spiegel innerer physikalischer

Tab. 7: Aufschlüsselung der 19 falsch negativen Differentialblutbilder am VCS (s. Tab. 6) nach Diagnose, prozentualer Abweichung zum visuellen Differentialblutbild, Angabe des \%-Bias (in Klammern), der jeweils ermittelten pathologischen Zellen im Ausstrich und Angabe der absoluten Leukozytenzahl (Abkürzungen: $m=$ männlich, $w=$ weiblich, Neutr. $=$ neutrophile Granulozyten, Eos. $=$ eosinophile .Granulozyten, Baso. = basophile Granulozyten, Mono. $=$ Monozyten, Lymph. $=$ Lymphozyten, MDS $=$ Myelodysplastisches Syndrom, $A M L=$ akute myeloische Leukämie, $C M L=$ chronisch myeloische Leukämie). Definition:

$\%$ Bias $=\frac{\text { (man. Diff. }- \text { VCS-Diff.) }}{\text { man. Diff. }} \times 100 \%$

\begin{tabular}{|c|c|c|c|c|c|c|c|c|}
\hline $\begin{array}{l}\text { Ifd. Nr. } \\
\text { (Alter, w, m) }\end{array}$ & Diagnose & \multicolumn{5}{|c|}{$\begin{array}{l}\text { prozentuale Abweichung } \Delta \text { (man. Diff - VCS-Diff.) } \\
\text { Werte in }()=\% \text { Bias }\end{array}$} & $\begin{array}{l}\text { pathologische } \\
\text { Zellen }\end{array}$ & $\begin{array}{l}\text { Leukozyten } \\
\quad(\mu \mathrm{l})\end{array}$ \\
\hline $1(77, m)$ & $\begin{array}{l}\text { retroperitoneales } \\
\text { Lymphom }\end{array}$ & $\begin{array}{c}-6 \\
(-9,8)\end{array}$ & $\underset{(33)}{2}$ & $\underset{(66)}{2}$ & 0 & $\begin{array}{l}-4 \\
(-36)\end{array}$ & 8 unreife Lymph. & 6000 \\
\hline $2(75, m)$ & aplastisches Syndrom & $\stackrel{2}{2}$ & $\begin{array}{c}3 \\
(33)\end{array}$ & 0 & $\begin{array}{l}-1 \\
(-25)\end{array}$ & $\begin{array}{c}-36 \\
(-600)\end{array}$ & $\begin{array}{l}34 \text { jugendl. atyp. } \\
\text { Lymphozyten }\end{array}$ & 3100 \\
\hline $3\langle 67, \mathrm{~m})$ & ALL & $\begin{array}{l}-4 \\
(-10)\end{array}$ & $\underset{(66)}{2}$ & $\begin{array}{c}1 \\
(100)\end{array}$ & $\begin{array}{l}-2 \\
(-28)\end{array}$ & $\begin{array}{l}-40 \\
(570)\end{array}$ & 43 unreife Lymph. & 3800 \\
\hline $4(58, w)$ & ALL, kompl. Remission & 0 & ${ }_{\varnothing}^{-2}$ & 0 & $\begin{array}{c}-9 \\
(-60)\end{array}$ & $\begin{array}{c}-4 \\
(-17,4)\end{array}$ & 16 unreife Lymph. & $900^{*}$ \\
\hline $5(62, w)$ & MDS & $\begin{array}{c}-2 \\
(-3,8)\end{array}$ & $\stackrel{2}{2}$ & 0 & $\begin{array}{c}-3 \\
(43)\end{array}$ & $\begin{array}{c}-1 \\
(-3,6)\end{array}$ & $\begin{array}{c}4 \text { unreife, atyp. } \\
\text { Zellen }\end{array}$ & 4300 \\
\hline $6(69, w)$ & $\mathrm{CML}$ & 0 & ${ }^{-1}$ & $0_{i}$ & $\begin{array}{c}-6 \\
(-40)\end{array}$ & $\begin{array}{c}2 \\
(8,7)\end{array}$ & 6 unreife Lymph. & 5300 \\
\hline $7(26, w)$ & Non Hodgkin Lymphom & $\begin{array}{l}-5 \\
(-12,5)\end{array}$ & 0 & $\begin{array}{c}2 \\
(50)\end{array}$ & $\begin{array}{c}-2 \\
(-67)\end{array}$ & $\begin{array}{c}-3 \\
(-6,8)\end{array}$ & $\begin{array}{l}17 \text { atypische } \\
\text { Zellen }\end{array}$ & $900^{*}$ \\
\hline $8(69, m)$ & ALL & $\begin{array}{c}-3 \\
(-6,7)\end{array}$ & 0 & 0 & $\begin{array}{c}1 \\
(25)\end{array}$ & 0 & $\begin{array}{l}4 \text { atypische } \\
\text { Lymph. }\end{array}$ & 1300 \\
\hline $9(73, w)$ & ALL & $\begin{array}{l}-4 \\
(-11,8)\end{array}$ & $\stackrel{-1}{\varnothing}$ & $\varnothing^{-1}$ & $\begin{array}{l}-5 \\
(-50)\end{array}$ & $\begin{array}{l}-20 \\
(-83)\end{array}$ & $\begin{array}{l}32 \text { atypische } \\
\text { Lymph. (blast.) }\end{array}$ & $800 *$ \\
\hline $10(74, m)$ & MDS & $\begin{array}{c}-1 \\
(-5,3)\end{array}$ & $\begin{array}{l}-1 \\
\varnothing\end{array}$ & 0 & $\begin{array}{c}-8 \\
(27)\end{array}$ & $\stackrel{1}{(2,4)}$ & $\begin{array}{l}10 \text { monozytoide } \\
\text { Zellformen }\end{array}$ & 3400 \\
\hline $11(69, w)$ & MDS, refraktäre Anämie & $\stackrel{7}{(17,1)}$ & 0 & 0 & $\begin{array}{c}-9 \\
(-69,2)\end{array}$ & $\begin{array}{c}-3 \\
(-7,7)\end{array}$ & 4 atyp. Zellen & 3200 \\
\hline $12(30, m)$ & Hodgkin Lymphom III & 0 & 0 & $\bar{\varnothing}^{-1}$ & $\begin{array}{c}-2 \\
(-14,3)\end{array}$ & $\begin{array}{c}-1 \\
(-6,7)\end{array}$ & 5 unreife Myelo. & 5900 \\
\hline $13(77, m)$ & lymphoblast. Lymphom & $\begin{array}{l}-9 \\
1-18\end{array}$ & $\begin{array}{l}-1 \\
(-20)\end{array}$ & $\begin{array}{c}1 \\
(100)\end{array}$ & $\begin{array}{c}1 \\
(8,3)\end{array}$ & $\begin{array}{c}1 \\
(3,7)\end{array}$ & $\begin{array}{l}5 \text { blastenähnl. } \\
\text { Zellen }\end{array}$ & 4600 \\
\hline $14(67, w)$ & Non-Hodgkin Lymphom & $\begin{array}{c}-6 \\
(-11,5)\end{array}$ & $\begin{array}{c}-1 \\
\varnothing\end{array}$ & $\bar{\emptyset}^{\circ}$ & $\begin{array}{c}-2 \\
(-40)\end{array}$ & $\stackrel{2}{(5,7)}$ & $\begin{array}{c}6 \text { blastenähnl. } \\
\text { Zellen }\end{array}$ & 6700 \\
\hline 15 (?) & Haarzelleukämie & $\begin{array}{l}-2 \\
(-5)\end{array}$ & 0 & 0 & $\begin{array}{c}-1 \\
(-50)\end{array}$ & $\begin{array}{l}-1 \\
(-1,9)\end{array}$ & 6 atypische Zellen & 4700 \\
\hline $16(68, m)$ & Hodgkin Lymphom & 0 & $\begin{array}{c}1 \\
(100)\end{array}$ & 0 & $\begin{array}{c}-1 \\
(-12,5)\end{array}$ & 0 & 5 atypische Zellen & 3100 \\
\hline $17(42, m)$ & CML & $\begin{array}{c}-6 \\
(-14,3)\end{array}$ & $\stackrel{1}{\varnothing}$ & 0 & 0 & $\begin{array}{l}0 \\
.\end{array}$ & 4 atypische Zellen & $700^{*}$ \\
\hline $18(73, w)$ & aplastisches Syndrom & $\begin{array}{c}-4 \\
(-13,8)\end{array}$ & $\underset{(33)}{2}$ & $\begin{array}{c}1 \\
(100)\end{array}$ & $\begin{array}{c}-3 \\
(-17,6)\end{array}$ & $\begin{array}{c}-3 \\
(-7,5)\end{array}$ & 5 atypische Zellen & 3200 \\
\hline $19(80, m)$ & AML & $\begin{array}{c}-2 \\
(-3,4)\end{array}$ & 0 . & $\begin{array}{l}-1 \\
\varnothing\end{array}$ & $\begin{array}{c}2 \\
(28,6)\end{array}$ & $\begin{array}{c}-8 \\
(-29,6)\end{array}$ & 8 atypische Zellen & 7600 \\
\hline
\end{tabular}


Ind chemischer Boschaffenheit der Zello und der Oberflächenlichtstreuung. Die Zelle wird im Gegensatz zum cytochemisch-cytometrischen Prinzip so wenig wie nötig verändert.

Ähnlich zum vorher beschriobenen Verfahren von Tochnicon werden auch beirn VCS die drei Meßgrößen einer Zelle in einem Scatterplot empirisch bestimmten Zellen des weißon Blutbildos zugeordnot. Auch hier hängen Genauigkeit der Differenziorung und clas Erkennen von pathologischen Veränderungen im Differentialblutbild in hohem Maße von der Gerätojustierung und von dem betriebenen Rechneraufwand ab.

Für die Geräte H 1 und H 6000 von Technicon liegen Vergleiche zwischon automatisierter und visueller Leukozytendifferenzierung vor (5-9). Bei der Evaluierung des $H 1$ von Teclınicon durch Thomas et al. (8) wurde ein ausgesuchtes Untersuchungsgut eingesetzt - Proben mit einer "Flagsetzung" am H 1 wurden nicht in die Evaluierung übernommen und "Ausreißer" bei der Messung wurden aus der Berechnung nach Passing/Bablok herausgenommen. Im Gegensatz dazu wurden in unserer Evaluierung des VCS alle Messungen in die statistische Auswertung einbezogen. Trotzdem erreicht man durchweg gute Korrelationen zum visuellen Differentialblutbild (Ausnahme: mäßige Korrelation bei Monozyten und fehlende Korrelation bei basophilen Granulozyten). Dieses Ergebnis bestätigt sich auch bei der Auswertung des Patientenkollektivs mit reaktiver Linksverschiebung. Linksverschiebungen werden vom VCS mit einer hohen Sensitivität von $99 \%$ angezeigt, und Zellpopulationen, die sich als bedeutsam für Verlaufskontrollen von Erkrankungen erwiesen haben, werden mit hoher Richtigkeit angezeigt.

Vergleichsstudien zwischen $\mathrm{H} 1$ und VCS von Katz et al: (11) ergaben eine gute bis sehr gute Korrelation bei den einzelnen Zellpopulationen mit Ausnahme der basophiIen Granulozyten, die vom VCS, Coulter um $50 \%$ niedriger als vom $H$ 1, Technicon angezeigt werden und einer relativen Monozytose ( $18 \%$ beim VCS). Die relative Monozytose spiegelt sich auch in den hier vorliegenden Daten wieder. Pathologische Veränderungen im Differentialblutbild werden vom VCS mit hoher Sensitivität angezeigt. Die Frage der Falsch-negativ-Rate bei pathologischen (reaktiv-linksverschobenen) Blutbildern liegt beim VCS bei etwa $1 \%$. Vergleiche von Katz et al. (11) und Klosson (12) bestätigen diese Ergebnisse. Die Anzahl der falsch positiven Ergebnisse wurde in dieser Arbeit nicht quantifiziert. Wie aus der Vergleichsstudie von Katz et al. zu entnehmen ist, liegt diese Rate sowohl beim VCS als auch beim $H 1$ bei etwa $15-20 \%$.

Beim Kollektiv der onkologischen Blutbilder (Kollektiv III) erreicht das VCS unter Berücksichtigung der absoluten Leukozytenzahlen eine Sensitivität von fast $85 \%$.

Review-Kriterien für das visuelle Nachdifferenzieren von mit dem VCS gemessenen Differentialblutbildern

Nachdifferenziert werden sollten:

- alle bekannten (und vermuteten) onkologischen Proben

- sporadische Kontrollen onkologischer Blutbilder (zur Therapie- und engmaschigen Verlaufskontrolle ist hingegen das VCS wegen seiner deutlich höheren Präzision besser geeignet)

- alle Blutbilder, in denen Zellpopulationswerte außerhalb der gängigen Richtwertbereiche differenziert werden - vom VCS indizierte Blutbilder, sofern es sich nicht um Kontrolluntersuchungen mit vorhandenen Vorwerten handelt
- Blutbilder mit pathologischen Gesamtleukozytenzahlen, sofern Leukozytenerhöhungen/-erniedrigungen bei Kenntnis der Grunderkrankung nicht erklärt werden können.

\section{Schlußfolgerung}

Beim VCS von Coulter handelt es sich um ein praxistaugliches Gerät, welches die anfangs gestellten Forderungen nach einer zuverlässigen Differenzierung von Zellen des normalen weißen Blutbildes erfüllt. Darüber hinaus werden vom VCS auch reaktive Linksverschiebungen, Eosinophilien und Monozytosen zuverlässig erkannt. Bei onkologischen Proben müssen mit der derzeitigen Software des VCS Abstriche gemacht werden. Wünschenswert wäre es, die Methodik so zu verfeinern, daß nicht nur onkologisch-morphologische Veränderungen zuverlässig erkannt werden, sondern auch unter Therapie (Cytostatika) veränderte Zellen zuverlässig und korrekt indiziert werden.

\section{Danksagung}

Die Autoren danken folgenden Damen für die technische Durchführung dieser Studie:

Frau K. Ehlhaus, Frau H. Hildebrand, Frau J. Middendorf, Frau U. Neuhaus, Frau U. Schacht, Frau A. Schulte, Frau K. Stecher, Frau C. Wrede und Frau S. Zeitler.

Ferner danken die Autoren Herrn Prof. Dr. H. Pralle für die Überlassung der onkologischen Blutbilder aus seiner Ambulanz, sowie Frau R. Stoy für die Kontrolldifferenzierung einiger hochpathologischer Blutbilder.

Schrifttum:

1. RÜMKE, C. L.: Die Zelldifferonzierung in Blutausstrichen: Variabilität der Ergebnisse. Triangle 4, 154-158 (1960).

2. RÜMKE, C. L., BEZEMER, P. D., KUIK, D. J.: Normal values and least significant differences for differential leukocyte counts. J. Chron. Dis. 28, 661-668 (1975).

3. RÜMKE, C. L.: The statistically expected variability in differential counting. In: Koepke, J. A., Differential leukocyte counting. S. 39. College of American Pathologists, Sokie, Illinois, 1979.

4. RASTETTER, J., NEIS, A., LANGE, H.-J.: Das Tochnikon Hemalog D System, Erfahrungen im Routinebetrieb. In: Auto-Analyzer Innovation Band I, 113-121, Edition Technicon 1978.

5. KAPLOW, L., ORLOWSKI, L., VAZNEILIS, M.: Evaluation of the Technikon $\mathrm{H} 6000$ hematology system. J. Clin. Lab. Automation 3, 167-174 (1983).

6. BREAKELL, E. S. MARCHAND, A., MARCUS, R. SIMSON, E.; Comparison of perfor6. BREAKELL, E. S., MARCHAND, A., MARCUS, R., SIMSON, E.: Comparison of performance for leukocyte differential counting of the Technikon $\mathrm{H} 6000$ System with
nual reference method using NCCLS standard. Blood Cells 11, 257-279 (1985).

nual roference method using NCCLS standard. Blood Cells 11, 257-279 (1985).
7. KATZ, N., LENZ, T.: Vergleich der Volumen-Verteilungsanalyse von Leukozyten am Coulter Stacker mit der cytochemisch-cytometrischen Klassifizierung am Technicon H 6000 und der mikroskopischen Differenzierung. Lab.med. 12, 354-362 (1988).

8. THOMAS, L., MÜLLER, Th., RODRIAN, J.: Vergleich der Bestimmung von Leukozytenpopulationen am Technicon $\mathrm{H} 1$ mit der mikroskopischen Differenzierung. Lab.med. 13, 213-219 (1989).

9. SMEETS, E. H. J., VAN WERSCH, J. W. J.: Performance of an three-part Dif. Impedance Cytometer (Sysmex E-4000) in Comparison with a Cytochemical Cytometer (Technicon H 6000). J. Clin. Chem. Clin. Biochem, 26, 531-540 (1988).

10. VAN WERSCH, J. W. J., BANK, C.: Zellzăhlung mit zusätzlicher Dreipopulations10. VAN WERSCH, J. W. J., BANK, C.: Zellzählung mit zusätzlicher Dreipopulationsund Fünfpopulationsdifferenzierung des peripheren Blutes:

11. MARTI, B., LENZ, T., KATZ, N.: Vergleich der automatisierten Blutbild-Ditferenzierung durch Streulicht-cytochemische $(H$ 1) und Streulicht-konduktometrische Analyse (VCS), Abstract in: Lab.mod. 13, 180 (1989).

12. KLOSSON, R. J.: Vergleich der Ergebnisse cytormetrischer, cytochemischer, konduktivmetrischer und mikroskopischer Verfahren zur Differenzierung der Leukozyten - ein Erfahrungsbericht, Abstract in: Lab.med. 13, 180-181 (1989).

13. BÜRGI, W. MARTI, H. R.: Automated blood count analysis by trimodal size distribution of leukocytes with the Sysmex E 5000, J. Clin. Chem. Clin. Biochem. 27, 365-368 (1989).

14. PASSING, H. BABLOK, W.: A new biometrical procedure for testing the equality of 14. PASSING, H., BABLOK, W.: A new biometrical procedure for testing the equality of measurements from two different analytical methods. Application of linear regression proced

15. EISENWIENER, H. G., BABLOK, W., BARDORFF, W., BENDER, R., MARKOWETZ, D., 15. EISENWIENER, H. G., BABLOK, W., BARDORFF, W., BENDER, R., MARKOWETZ, D.,
PASSING, H., SPAETHE, R., SPECHT, W., VOLKERT, E.: Statistische Auswertung beim Methodenvergleich. Lab.med. 8. 232-244 (1984).

16. HAECKEL, R., PASSING, H.: Statistische Probleme beim Vergleich klinisch-chemischer Analysenverfahren. J. Clin. Chem. Clin. Biochem. 23, 307-318 (1985).

17. Int. Pat. Class. 4, G 01N 33/48 voim 22. 9. 88.

Anschrift für die Verfasser:

Prof. Dr. H. Schriewer

Zentrallaboratorium des Kreiskrankenhauses

Paulmannshöherstraße 14

5880 Lüdenscheid 